\title{
Piecing together the puzzle of cutaneous mosaicism
}

\author{
Amy S. Paller \\ Department of Dermatology, Feinberg School of Medicine, Northwestern University, Chicago, Illinois, USA.
}

\begin{abstract}
Autosomal dominant disorders of the skin may present in a pattern following the lines of embryologic development of the ectoderm. In these cases, the surrounding skin is normal, and molecular studies have shown that the causative mutation is confined to the affected ectodermal tissue (type 1 mosaicism). Rarely, an individual shows skin lesions that follow the pattern of type 1 mosaicism, but the rest of the skin shows a milder form of the disorder (type 2 mosaicism). A new study provides the molecular basis for type 2 mosaicism (see the related article beginning on page 1467).
\end{abstract}

More than a century ago, the lines of Blaschko were first described by German dermatologist Alfred Blaschko, based on the observation of patterned skin lesions that were linear on the extremities, S-shaped on the anterior trunk, and V-shaped on the back (1) (Figure 1). Blaschko's lines are thought to trace pathways of ectodermal cell development. Happle has championed the concept that the cutaneous lesions following Blaschko's lines are manifestations of somatic mosaicism (2).

Mosaicism results when a postzygotic mutation occurs in gonadal (germline mosaicism) or other (somatic mosaicism) cells, leading to an organism with two or more genetically different populations of cells that originate from a genetically homogeneous zygote. In most cases of clinical mosaicism, the linear patterns of abnormal skin are surrounded by normal skin; this has been termed type 1 mosaicism. The first demonstration that clinical mosaicism in skin along Blaschko's lines correlates perfectly with keratinocyte genetic mosaicism was based on the discovery in 1994 of keratin 10 mutations in lesional skin, but not in clinically normal skin, of patients with a form of epidermal nevi that histologically shows epidermal lysis and marked stratum corneum thickening (3). These are characteristic features of the skin of patients with epidermolytic hyperkeratosis, a genetic condition with diffuse bilateral involvement that is known to result from mutations in keratin 10 (4).

Conflict of interest: The author has declared that no conflict of interest exists.

Citation for this article: J. Clin. Invest. 114:1407-1409 (2004). doi:10.1172/JCI200423580.
The molecular basis for somatic mosaicism in several other epidermal disorders that follow Blaschko's lines has since been established, providing further evidence that Blaschko's lines represent pathways of ectodermal embryonic development (5-8). Mosaicism has also been shown to reverse clinical phenotypes, particularly in patients with forms of the group of generalized blistering disorders known as epidermolysis bullosa $(9,10)$. The localized genetic correction of keratin 14 in basal keratinocytes in some of these patients leads to progressive clinical improvement, which reflects the selective advantage of normal versus lesional proliferating keratinocytes that has recently been shown in mouse models (11).

\section{Two types of mosaicism}

Several patients with autosomal dominant disorders of the skin have now been described as showing diffuse cutaneous involvement with their genetic disease but linear patterns of exacerbation of the skin disorder that follow Blaschko's lines, although this is a rare occurrence. This type 2 form of mosaicism has been seen (a) in patients with superficial actinic porokeratosis with streaks of thick linear porokeratosis $(12,13)$; (b) in those with tumor syndromes in which large numbers of cutaneous tumors are confined to a segmental or linear distribution, e.g., dermal neurofibromas in neurofibromatosis type 1 (14), leiomyomas in multiple cutaneous and uterine leiomyomatosis (15), and hamartomas of the tongue in tuberous sclerosis (14); and (c) in individuals with Hailey-Hailey disease, characterized by segmental areas of severe crusting, ooz- ing, and erythema in addition to the symmetrically distributed milder plaques of erythema and crusting (16).

\section{Providing a molecular mechanism to explain type $\mathbf{2}$ mosaicism}

In this issue of the JCI, Poblete-Gutierrez et al. (17) provide evidence of the molecular basis of type 2 mosaicism in HaileyHailey disease. This autosomal dominant disorder, characterized by abnormal keratinocyte differentiation, results from mutations in ATP2C1, which encodes the calcium pump protein ATPase, $\mathrm{Ca}^{2+}$ transporting, type $2 \mathrm{C}$, member 1 . In contrast to many autosomal dominant disorders of the skin in which lesions are widespread and, upon clinical examination, no skin appears to be spared, the red, crusted plaques of Hailey-Hailey disease tend to be symmetrically distributed primarily at intertriginous areas, where opposing skin surfaces may touch and rub, such as skin folds of the groin, underarm, and breast. The patient studied by Poblete-Gutiérrez et al. developed widely distributed, severely erythematous, and crusted plaques along Blaschko's lines on the left side of her body $(16,17)$. In contrast to the typical onset of plaques largely confined to intertriginous areas in late childhood or young adulthood, these extensive plaques were first noted when the patient was 3 months of age (17).

Keratinocytes isolated by laser dissection or cultured after biopsy of these severely affected areas harbored a homozygous genetic defect in ATP2C1, while keratinocytes isolated from other sites showed the heterozygous defect. In this patient with type 2 mosaicism, the loss of heterozygosity resulted from mutation and loss of the paternal allele. The localized genetic change from one normal allele and one abnormal allele (heterozygous state of a typical individual with Hailey-Hailey disease) to only the abnormal allele (loss of heterozygosity) gave the patient a double dose of the mutant gene in severely affected areas, 
A

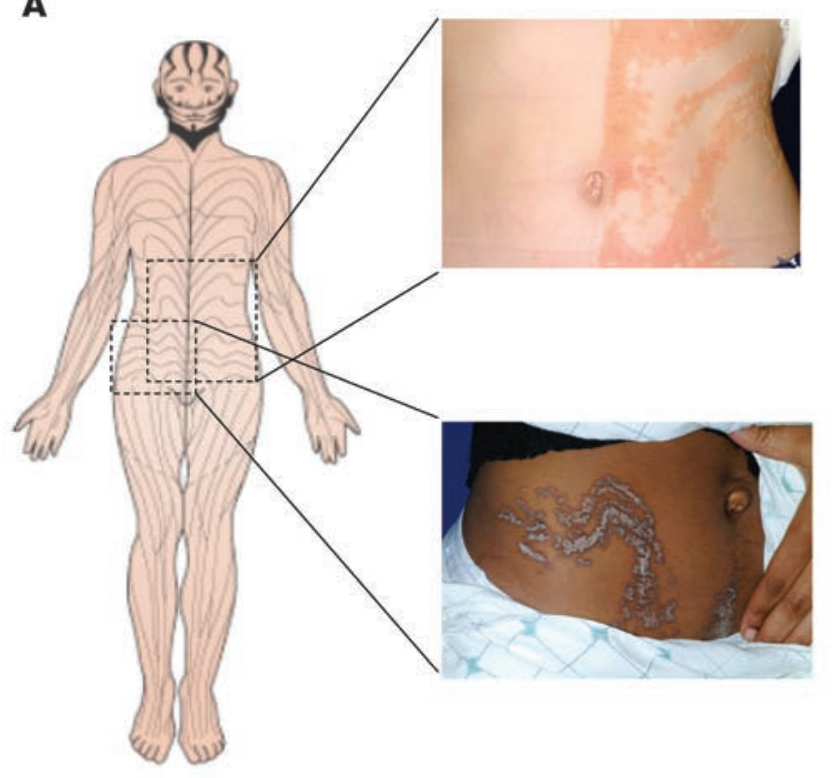

B

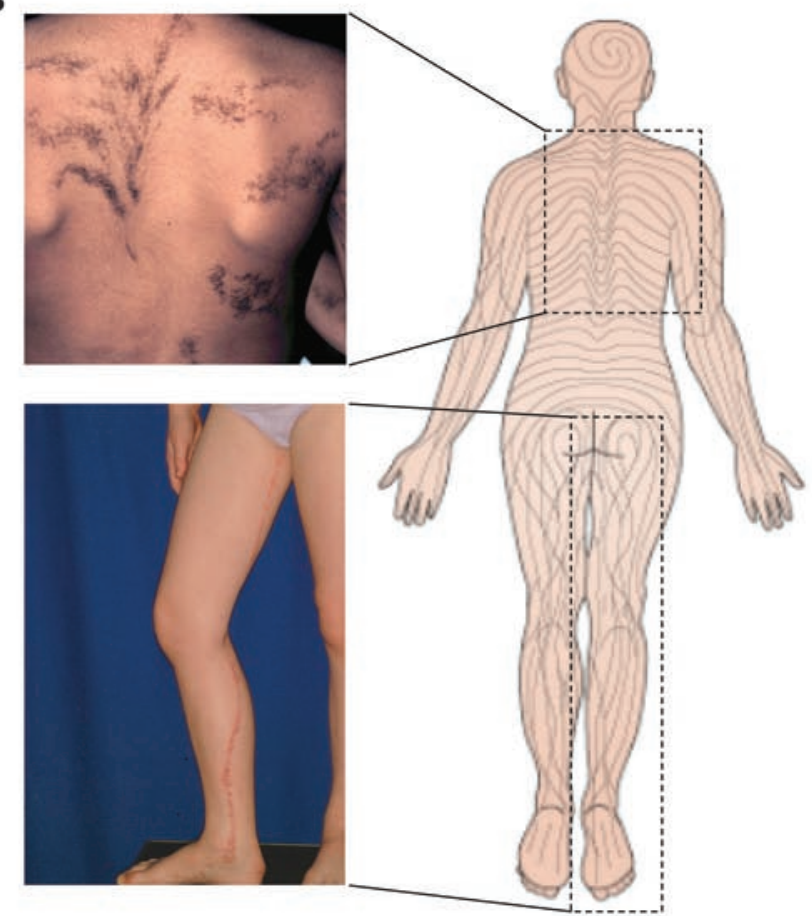

\section{Figure 1}

The lines of Blaschko, originally described in 1901, are thought to trace the pathway of ectodermal cell development. These lines are linear on the extremities, S-shaped on the anterior trunk, and V-shaped on the back. Epidermal nevi are examples of cutaneous mosaicism in which the localized thickening of the epidermis is patterned along the lines of Blaschko. This figure illustrates several forms of epidermal nevi and the correlation of their orientation with the lines of Blaschko. A shows two verrucous (or keratinocytic) forms of epidermal nevus on the anterior (upper image) and anterolateral (lower image) regions of the trunk that correlate nicely with the lines of Blaschko, shown schematically. Similarly, B shows epidermal nevi of the epidermolytic hyperkeratotic type (upper image) in a curvilinear configuration and an inflammatory linear verrucous form of epidermal nevus on the leg (lower image). The molecular mechanism underlying the defect is known for only one of these clinical manifestations, the epidermolytic hyperkeratotic form of epidermal nevus. The 6-year-old girl shown in B showed a missense mutation in keratin 10 in cultured keratinocytes from lesional, but not normal-appearing, skin.

leading to the patterned disease exacerbation along Blaschko's lines. A variety of mutational events may occur that lead to loss of the normal allele (hemizygosity) or duplication of the mutant chromosome (homozygosity), among them point mutation, localized gene conversion, mitotic recombination, deletion, and nondisjunction. In the patient with type 2 mosaicism and Hailey-Hailey disease, Poblete-Gutiérrez et al. demonstrate loss of the paternal allele with duplication of the mutated maternal allele, probably by postzygotic mitotic recombination (17).

The novel discovery of the basis for type 2 mosaicism contributes to our understanding of gene mosaicism as well as of embryologic development of the ectoderm along the defined lines of Blaschko. However, demonstration of the correlation of mosaic genotype and phenotype in these studies and in the studies of the molecular basis for type 1 mosaicism should be seen as early investigations in the understanding of gene mosaicism. One continuing area of investigation is the relationship of the extent of cutaneous manifestation of mosaicism to the risk of germline involvement, which could lead to a widespread skin disorder in offspring of a parent with type 1 mosaicism. Keratinocyte mutations with an earlier time of onset during the postzygotic period are theorized to be associated with a lower risk of germline mutation, and more extensive skin involvement is thought to be associated with a higher risk. In type 1 mosaicism, up to $50 \%$ of peripheral blood leukocytes may harbor the mutation; given the mesodermal origin of both blood cells and germline cells, one might surmise that a higher ratio of mutant to normal genomic DNA would be associated with a higher risk of germline involvement, but this possibility has not been assessed. Use of engineered mouse models of cutane- ous mosaicism with inducible localized mutations may allow us to correlate the extent and distribution of mosaic manifestations with the time at which these postzygotic mutations occur and to correlate this timing with ectodermal versus mesodermal development.

Importantly, even less is known about the embryologic development and mosaicism of nonectodermal structures. Two examples of possible dermal mosaicism are morphea (a disorder of localized dermal sclerosis) and focal dermal hypoplasia (characterized by streaks of dermal tissue hypoplasia, often in association with streaks in the metaphyses of long bones). However, the underlying genetic basis for these disorders remains unknown, preventing the confirmation of mutation within cells of mesodermal origin. Several studies have clearly demonstrated that the genotypes of keratinocytes and underlying fibroblasts do not correlate in mosaic 
skin conditions, confirming the different developmental patterns of ectoderm and mesoderm (3). The disparate development of ectodermal and mesodermal structures emphasizes the need to study affected tissues when seeking evidence of gene mosaicism. Patterned epidermal and dermal mosaic disorders (such as morphea and focal dermal hypoplasia) provide an ideal focus for future investigations that will elucidate the mechanisms of embryologic development of both ectodermal and mesodermal tissues.

Address correspondence to: Amy S. Paller, Department of Dermatology, 645 North Michigan Avenue, Suite 520, Chicago, Illinois 60611, USA. Phone: (312) 695-0197; Fax: (312) 695-0664: E-mail: apaller@northwestern.edu.

1. Blaschko, A. 1901. Die Nervenverteilung in der Haut in ibrer Beziehung zu den Erkrankungen der Haut. Wilhelm
Braunmuller. Vienna, Austria and Leipzig, Germany.

2. Happle, R. 1993. Mosaicism in human skin. Understanding the patterns and mechanisms. Arch. Dermatol. 129:1460-1470.

3. Paller, A.S., et al. 1994. Genetic and clinical mosaicism in a type of epidermal nevus. N. Engl. J. Med. 331:1408-1415.

4. Rothnagel, J.A., et al. 1992. Mutations in the rod domains of keratins 1 and 10 in epidermolytic hyperkeratosis. Science. 257:1128-1130.

5. Colman, S.D., Rasmussen, S.A., Ho, V.T., Abernathy, C.R., and Wallace, M.R. 1996. Somatic mosaicism in a patient with neurofibromatosis type 1 . Am. J. Hum. Genet. 58:484-490.

6. Munro, C.S., and Wilkie, A.O. 1998. Epidermal mosaicism producing localised acne: somatic mutation in FGFR2. Lancet. 352:704-705.

7. Sakuntabhai, A., Dhitavat, J., Burge, S., and Hovnanian, A. 2000. Mosaicism for ATP2A2 mutations causes segmental Darier's disease. J. Invest. Dermatol. 115:1144-1147.

8. Terrinoni, A., et al. 2000. A mutation in the V1 domain of K16 is responsible for unilateral palmoplantar verrucous nevus. J. Invest. Dermatol. 114:1136-1140.

9. Jonkman, M.F., et al. 1997. Revertant mosaicism in epidermolysis bullosa caused by mitotic gene conversion. Cell. 88:543-551.

10. Smith, F.J., Morley, S.M., and McLean, W.H. 2004.
Novel mechanism of revertant mosaicism in Dowling-Meara epidermolysis bullosa simplex. J. Invest. Dermatol. 122:73-77.

11. Arin, M.J., Longley, M.A., Wang, X.J., and Roop, D.R. 2001. Focal activation of a mutant allele defines the role of stem cells in mosaic skin disorders. J. Cell Biol. 152:645-649.

12. Happle, R. 1991. Somatic recombination may explain linear porokeratosis associated with disseminated superficial actinic porokeratosis. Am.J. Med. Genet. 39:237.

13. Happle, R. 1996. Segmental forms of autosomal dominant skin disorders: different types of severity reflect different states of zygosity. Am. J. Med. Genet. 66:241-242.

14. Itin, P.H., and Buechner, S.A. 1999. Segmental forms of autosomal dominant skin disorders: the puzzle of mosaicism. Am. J. Med. Genet. 85:351-354.

15. Smith, C.G., Glaser, D.A., and Leonardi, C. 1998. Zosteriform multiple leiomyomas. J. Am. Acad. Dermatol. 38:272-273.

16. Vakilzadeh, F., and Kolde, G. 1985. Relapsing linear acantholytic dermatosis. Br. J. Dermatol. 112:349-355

17. Poblete-Gutiérrez, P., et al. 2004. Allelic loss underlies type 2 segmental Hailey-Hailey disease, providing molecular confirmation of a novel genetic concept. J. Clin. Invest. 114:1467-1474. doi:10.1172/ JCI200421791.

\title{
The multiple causes of human SCID
}

\section{Rebecca H. Buckley}

Department of Pediatrics, Duke University Medical Center, Durham, North Carolina, USA.

\begin{abstract}
SCID, a syndrome characterized by the absence of T cells and adaptive immunity, can result from mutations in multiple genes that encode components of the immune system. Three such components are cytokine receptor chains or signaling molecules, five are needed for antigen receptor development, one is adenosine deaminase - a purine salvage pathway enzyme, and the last is a phosphatase, CD45. In this issue of the JCI, a report describes how complete deficiency of the $C D 3 \varepsilon$ chain of the $T$ cell antigen receptor/CD3 complex causes human SCID (see the related article beginning on page 1512).
\end{abstract}

Human SCID was first reported by Glanzmann and Riniker in 1950 (1). Swiss infants with the condition were profoundly lymphopenic and died of infection before their first or second birthdays. In the ensuing years, differences were noted in inheritance patterns for SCID. This indicated that there was more than one cause for this fatal syndrome characterized by an absence of $T$ cells and all adaptive immunity. In many families there was an X-linked recessive mode of inheritance while in others an autosomal recessive mode of inheritance was observed. The first discovered molecular cause of human

Conflict of interest: The author has declared that no conflict of interest exists.

Citation for this article: J. Clin. Invest. 114:1409-1411 (2004). doi:10.1172/JCI200423571.
SCID, adenosine deaminase deficiency, was reported in 1972 (2). However, it was not until 21 years later, in 1993, that a second fundamental cause of the condition was found, i.e., the molecular basis of X-linked human $\operatorname{SCID}(3,4)$. Over the past 11 years, remarkable progress has been made in elucidating several other causes of this syndrome (5). Advances in molecular biology and the Human Genome Project as well as increased knowledge of various components of the immune system through studies of mutant mice and humans with genetically determined immunodeficiencies have all contributed to this understanding. It is now known that SCID can be caused in humans by mutations in at least 10 different genes (Table 1) (6-11), and the likelihood is that there are other causes yet to be discovered. The gene prod- ucts of 3 of these mutated genes are components of cytokine receptors (the IL-2 receptor $\gamma$ chain that is also shared with 5 other cytokine receptors [IL-4R, IL-7R, IL-9R, IL-15R, and IL-21R], JAK3, the primary signal transducer from the common $\gamma$ chain, and the $\alpha$ chain of the IL-7 receptor); the products of 5 more genes (RAG1, RAG2, Artemis, CD38, and CD3e) are necessary for antigen-receptor development;

\section{Table 1}

Ten abnormal genes in human SCID

Cytokine-receptor genes
IL-2RG
JAK3
IL-7R $\alpha$
Antigen-receptor genes
RAG1
RAG2
Artemis
CD3ठ
$C D 3 \varepsilon$
0ther genes
ADA
$C D 45$

